\title{
Role of Exponential and Power Law Formulations in Contact Stress
}

\author{
Vahid Monfared ${ }^{1 *}$, Mohammadhassan Hassan ${ }^{2}$, Saeed Daneshmand ${ }^{3}$, Farshad Taheran ${ }^{4}$ and \\ Amirhossein Monfared ${ }^{5}$ \\ 'Department of Mechanical Engineering, Zanjan Branch, Islamic Azad University, Zanjan, Iran; \\ vahid.monfared@iauz.ac.ir \\ 2Department of Mechanical Engineering, Sharif University of Technology, PO Box 11365-9567, Tehran, Iran; \\ mh.hasan.1990@gmail.com \\ 3Department of Mechanical Engineering, Majlesi Branch, Islamic Azad University, Isfahan, Iran; \\ s.daneshmand@iaumajlesi.ac.ir \\ ${ }^{4}$ School of Mechanical Engineering, Sharif University of Technology, Tehran, Iran; F_Taheran@mech.sharif.edu \\ ${ }^{5}$ Department of Industrial Intelligence Research Group. ACECR, Zanjan Branch, Iran; \\ monfared.amirhossein@yahoo.com
}

\begin{abstract}
Analyzing contact stress in rails and wheels are very important in mechanical and railway engineering. In this paper, new formulations of the contact stress are presented for two rolling bodies by exponential and power law forms semianalytically. Innovative elastic wheel-rail contact models and FE Modeling are proposed. The present model assumes the collection of rail and wheel as elastic deformable bodies and needs numerical and novel analytical solutions. Results of this work are close to the Hertz Stress, previous published work and FEM results, in which good agreements are found among the results. So, we can rely on this method and their results. With this approach, suitable results will be achieved. Important novelty of this research is presentation of new analytical formulations in the Power Law (PL) and Exponential Forms (EF) for obtaining contact stress in the rolling bodies.
\end{abstract}

Keywords: Contact Stress, Exponential and Power Law Forms, Hertz's Elliptic Rolling Bodies.

\section{Introduction}

Physics and Mechanics of the collection of the wheel-rail contact is one of the fundamental and essential entities for investigation in railway engineering, requiring both: enormous application skill and reliable analysis approaches. Theoretical models depicting the physics of this fact are only defined for specific type of simple contact geometries, hence for more complex geometries the analytical models employing closed forms remain hard to grasp. Railway engineers successfully applied one of the numerical computation methods known as Finite Element Method (FEM) or Simple Direct Formulations into rail-wheel contact problems to verify their results by comparing them to their actual life information determined in previous years. Determination of the contact surfaces requires knowledge of some geometric constants used in the formulation in the rolling bodies. Two solid discs and rolling body are put together to generate an elliptic contact surface. The wheel-rail contact can be explained by the general case of an elliptic contact surface. Although there are some important simplifications that can be applied in modeling, other formats of contact surfaces are not developed here. Evaluating surface and subsurface stresses requires the determination of the displacements field. One also needs to calculate the contact surface.

*Author for correspondence: 
Contact and the damage of the rail and wheel to both surfaces are of great interest to railway engineers. Understanding various loading methods is the first step in recognizing the root cause of such problems. Contact stresses are significant when contact is not fixed but cyclic in nature such as locomotive wheel-rail road rail.

In order to study the stress analysis, factors of critical stress must be determined. One of the frequent flaws and defects of rails which lead to their failure and fracture is a vertical crack on the end of the rails. This is because loading will make a tensile and compressive stress on the end of the rail. Therefore, these stresses with the tensile and compressive stresses will cause compressive and tensile stresses on the end of rail respectively and these lead to the growth of vertical crack at the end of the rail, and eventually cause failure and fracture in it.

Significant researches were performed over the last years to investigate contact stress, its fracture and fatigue crack growth, laws in rolling bodies etc., Some contact stress problems, stress distribution on elliptical contact surface and classical Hertz contact problems have been studied by many researchers analytically ${ }^{1-4}$.

Contact between elastic bodies with and without creep has been investigated by Smith and Liu ${ }^{1}$.

These models could be applied only to rectangular contact surface. They diminished the equations to determine the normal and shear stresses theoretically, both in the contact surface and inside the bodies. They used the maximum value for tangential force, as described by Coulomb's Law. They analyzed stresses because of tangential and normal loadings on an elastic solid applying to several contact stress problems ${ }^{1}$. Contact stress distribution on elliptical contact surfaces under radial and tangential forces has been presented. These results have been compared with photo elasticity experiments and have been found to be in good agreement ${ }^{2}$. Existing theories have been assembled ${ }^{3}$ for circular and rectangular contact surface, for which both the longitudinal and tangential loadings were considered. They presented a model to determine the stress field for both cases and some helpful results in the conventional Hertz contact problem. In what follows, some experimental attempts were performed by some investigators ${ }^{5-7}$. For example, failure estimation diagram was studied ${ }^{6}$ for components under rolling contact loading. Experimental and numerical modelling of wheel-rail contact and wear was presented by Roviraa et al. .

Moreover, numerical studies have been carried out ${ }^{8-13}$ for analyzing contact stress between rail and wheel by finite element methods. The results obtained were similar to the ones determined by previous researchers. Analysis of wheel-rail interaction was investigated using FE software by Sladkowski and Sitarz ${ }^{8}$.

3D Elastoplastic stress analysis of the rail-wheel rolling contact was presented by Wen et al. ${ }^{11}$. Arslan and Kayabasi ${ }^{13}$ studied 3-D rail-wheel contact analysis by FEA.

Additionally, several studies have been performed regarding engineering and mathematical problems, which can be of great help for the investigators ${ }^{14-20}$. Analyzing previous works shows that, most of the works in contact stress problems in rail-wheel contact have been performed by FEM numerically.

Priority is given for the determination of critical surface of stress and also, positions of critical compressive and tensile stress can be determined by FEM (Figures 1,2). Figure 2 shows contact surface and analysis of contact forces in the rail and wheel. The Hertz Stress must be almost the same as the stress in the y direction.

The interesting part is the determination of the new contact stress formulations in two rolling bodies, where

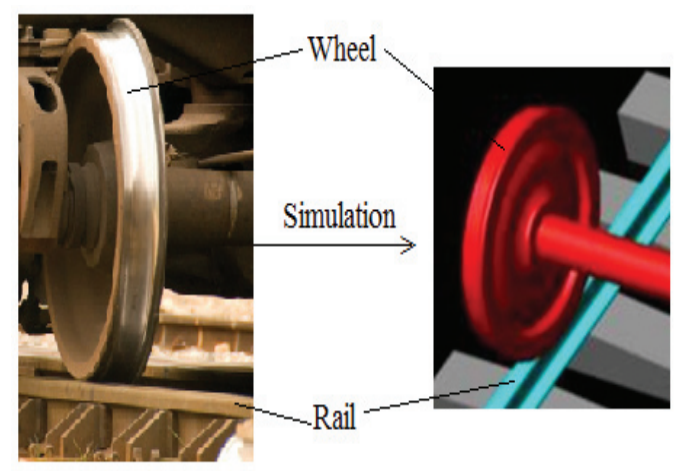

Figure 1. Position of contact of the wheel and rail.

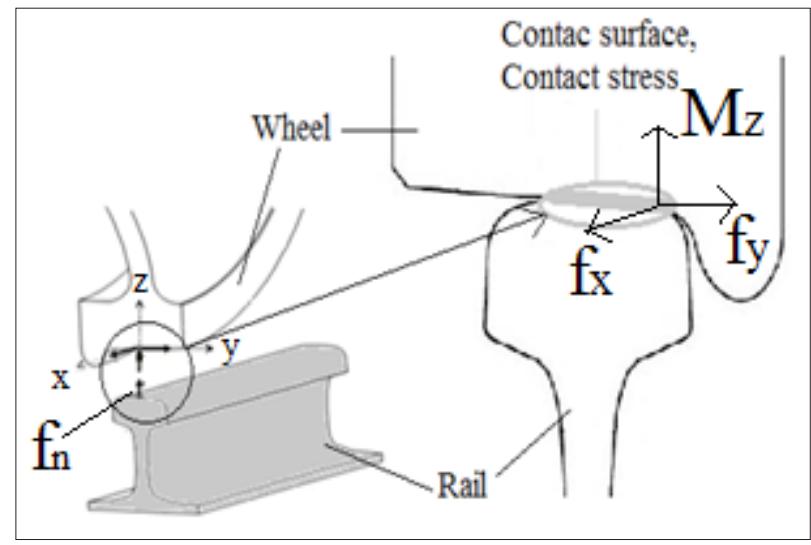

Figure 2. Contact of the wheel and rail in direct path. 
these results are equal to the Hertz Stress, FEM and previous published results. This new formulation doesn't require any additional parameters for determination of the contact stress in the rolling bodies. The number of parameters for calculating contact stress is lesser than other formulations and ordinary and also simple calculation is their added point. It is also easy and also user friendly. In addition, the new exponential and power law formulation results are similar and coincide with the FEM results. For verifying the new formulations results, these results have been compared with the previous ${ }^{9-12}$ research results. Therefore, an important and main objective of this research is to find the contact stress (with critical regions) in any place of the wheels and rails near the contact region in different mathematical forms analytically. One of the important advantages of this study is its simplicity unlike the complex procedure of the previous researches. In this paper, exponential and power law formulations are presented for analyzing contact stress in two rolling bodies. The main purpose of this work is to analyze the contact stress based on Power and Exponential laws.

\section{Materials and Methods}

Stress distribution is a significant reality or a collection of wheel-rail contact interfaces, which are dependent on geometry and physics of the contact areas, load conditions and material properties of the system of interest. Computation of these stresses becomes lot more intricate for 3 Dimensional (3D) actual life geometries. For early failure and fracture, the size of the crack must be about 6 centimeters, so the area of the crack will be larger than the end surface of the rail. Standard UIC60 are used for stress analysis (Figure 3).

Figure 4 shows meshed and unmeshed contact area and region of rail-wheel collection in contact state generally.

Hertz's formulation presents just one method to contact stress theory, its success in approximation of permissible maximum or desired minimum local loads warrants a comprehensive examination of its features. A primarily convincing argument is its role in the optimization of design and use of ball and roller bearings. Hertz stress formulation is extensive, powerful and a primitive formula which is used to determine the contact stress for curved bodies, and so on.

In this paper, new power law and exponential formulation of contact stress for two rolling bodies are presented,

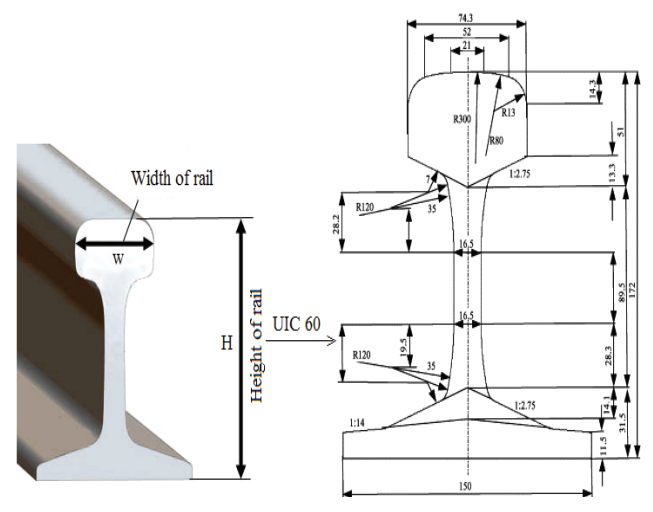

Figure 3. Position of contact for UIC60.

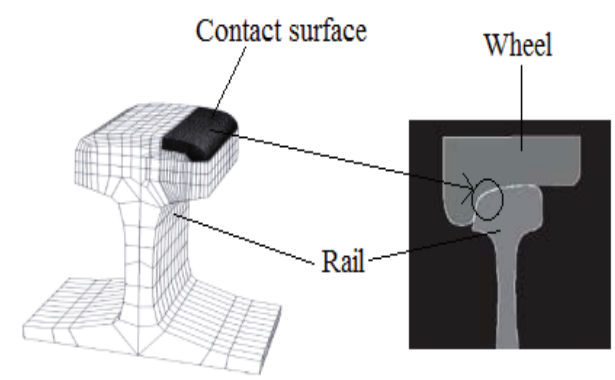

Figure 4. Direction of loading in contact surface.

and their results are close to Hertz Stress-Formulation and FEM results.

These formulations are determined by the concept of contact and normal stress and numerical results, as well as, constants in these methods are determined by analytical and numerical results. In these methods, contact stress relates with force, elastic module and rectangular contact surface. This can be seen in Equations 1-8:

$$
\sigma_{\max }=f\left(F, E, A_{\text {rect }}\right)
$$

Exponential form,

$$
\sigma_{\max } \propto \frac{E \times A_{\text {rect }}^{2} \times \operatorname{Exp}\left(F A_{\text {rect }}^{3}\right)}{K_{1}}
$$

Power Law form,

$$
\sigma_{\max } \propto \frac{E \times F \sqrt[5]{A_{\text {rect }}^{2}}}{K_{2}}
$$

Also from (Monfared ${ }^{12}$, we have:

$$
\sigma_{\max } \propto\left(\frac{F \times E}{A_{\text {rect }}}\right)^{\frac{1}{2}} \times K_{3}
$$




$$
\begin{gathered}
A_{\text {rect }}=a \times b \\
\sigma_{\text {max }}=\left(\frac{F \times E}{A_{\text {rect }}}\right)^{\frac{1}{2}} \times \sqrt{6.78} \times 10^{-7}
\end{gathered}
$$

where $k_{\mathrm{i}}^{\prime} s$, are constant coefficients for the proportion of equation. With attention to analytical and numerical results with FEM's assumptions, the values of $k_{\mathrm{i}}^{\prime} s$, are equal to $0.2 \times 10^{7}, 10^{10}, \sqrt{6.78} \times 10^{-7}$ respectively. That is,

$$
\begin{gathered}
\sigma_{\max }(M P a)=\frac{E \times A_{\text {rect }}{ }^{2} \times \operatorname{Exp}\left(F A_{\text {rect }}{ }^{3}\right)}{0.2 \times 10^{7}} \\
\sigma_{\max }(M P a)=\frac{E \times F \sqrt[5]{A_{\text {rect }}{ }^{2}}}{10^{10}}
\end{gathered}
$$

Thus, maximum stress values are also determined by eq. 8 . In the following section, results and discussions are presented for more clarification.

\section{Results and Discussions}

In this section, new formulation and FEM results are presented and will be compared with previous results ${ }^{12}$, where $\mathrm{F}$ is force in kilo-Newton, elastic module $\left(\frac{N}{\mathrm{~m}^{2}}\right)$, and $A_{\text {rect }}$ is rectangular contact surface $\left(\mathrm{m}^{2}\right)$. Values of the hertz stress and new formulation are compared in Figure 5 and Table 1 . The loading in this simulations are 90, 95, 100, 105, $110 \mathrm{KN}$.

Table1 shows that the difference of the results is very small. According to Figure 5 and Table 1, the results of hertz stress are the same as the new formulation for contact stress ${ }^{12}$.

The new formulation as well as simplicity, requires a few data for the determination of contact stress. Finite Element Model (FEM) of rail and wheel are shown in Figures 4 and 11. This 3D model geometry is assigned to the ANSYS Software and meshed with SHELL-63, SOLID-45, 8-noded, hex elements as shown in Figure 11. Contact between Rail and Wheel is modeled using ANSYS Contact 173 elements placed on wheel and rail. FEM mesh (wheel and rail) is obtained having a total of 35740 elements and 22830 nodes.

It demonstrates that the number of data for deter-

\begin{tabular}{|c|c|c|c|c|c|}
\hline $\begin{array}{l}\text { Stress } \\
(\mathrm{MPa})\end{array}$ & $\begin{array}{c}90 \\
(\mathrm{KN})\end{array}$ & $\begin{array}{c}95 \\
(\mathrm{KN})\end{array}$ & $\begin{array}{c}100 \\
(\mathrm{KN})\end{array}$ & $\begin{array}{c}105 \\
(\mathrm{KN})\end{array}$ & $\begin{array}{c}110 \\
(\mathrm{KN})\end{array}$ \\
\hline $\begin{array}{l}\text { Exponential } \\
\text { form }\end{array}$ & 194.34 & 199.66 & 205.08 & 210.65 & 216.37 \\
\hline Power law & 192.72 & 197.98 & 203.1 & 208.1 & 212.97 \\
\hline $\begin{array}{l}\text { Hertz } \\
\text { stress }\end{array}$ & 192.51 & 197.78 & 202.92 & 207.93 & 212.82 \\
\hline $\begin{array}{l}\text { New formu- } \\
\text { lation }^{12}\end{array}$ & 192.51 & 197.79 & 202.92 & 207.94 & 212.83 \\
\hline $\begin{array}{l}\text { FEM } \\
\text { (a quarter } \\
\text { of elliptic } \\
\text { surface) }\end{array}$ & 191 & 196 & 201 & 207 & 211 \\
\hline $\begin{array}{l}\text { FEM (half } \\
\text { of elliptic } \\
\text { surface) }\end{array}$ & 189 & 191 & 199 & 201 & 206 \\
\hline $\begin{array}{l}\text { FEM (three } \\
\text { fourth of } \\
\text { elliptic } \\
\text { surface) }\end{array}$ & 184 & 188 & 197 & 195 & 200 \\
\hline $\begin{array}{l}\text { FEM (a sixth } \\
\text { of elliptic } \\
\text { surface) }\end{array}$ & 196 & 198 & 206 & 208 & 215 \\
\hline $\begin{array}{l}\text { FEM (one } \\
\text { eighth of } \\
\text { elliptic } \\
\text { surface) }\end{array}$ & 198 & 202 & 208 & 209 & 219 \\
\hline
\end{tabular}
mination of contact stress in new formulation is lesser
Table 1. Comparison of hertz, new formulation ${ }^{12}$, FEM, exponential and power law stresses

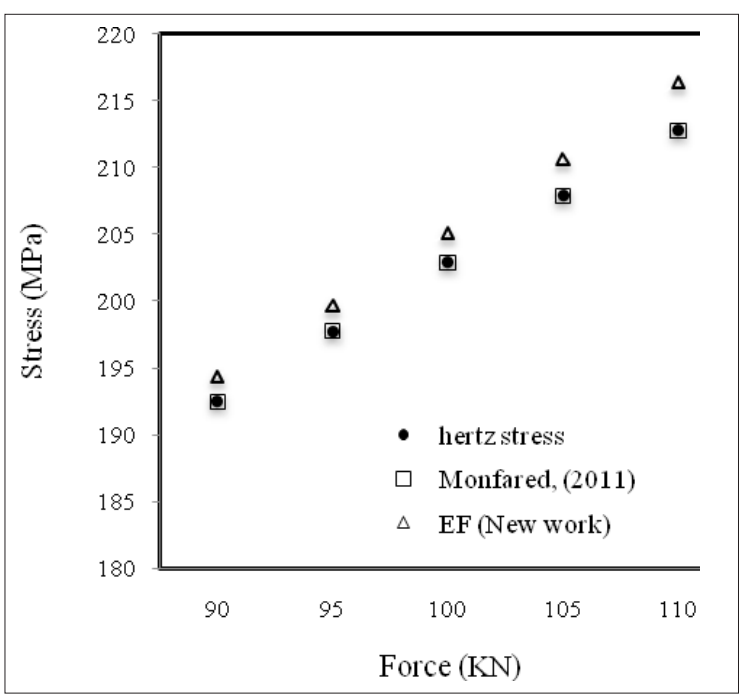

Figure 5. Comparison of hertz stress, new contact stress formulation $^{12}$ and Exponential Form (EF). 
than the hertz stress formulation. In order to analyze the contract stresses, Hertz stress and numerical methods are used. The induced and applied loads in the contact surface have been performed by FEM analysis. Figures 3 and 4 show that the standard UIC60 and direction of forces in the contact surface. In this section, we will analyze the contact stress, critical points and stress, and the beginning of the failure in the rail for the straight path. For this purpose we induce the static load to the wheel and rail by FEM and then analyze the stress. The induced load to one wheel is about 9 to 11 tones. Then we determine hertz stress and compare it with stress in y direction, which generally these results should be equal in every respect and also contact surface is an elliptical surface approximately.

In this research, the axial load is considered about 20 tones. The area of contact surface is assumed about 1 $\mathrm{cm}^{2}$. The loads that are induced to the rail and the wheel are 90, 95, 100, 105 and $110 \mathrm{KN}$. Finally, the results of three methods are the same if the area of contact is one third of the area. Figures 5, 6, 7, 8, 9 and 10 show the results of three methods and their comparisons. For modeling of the wheel and the rail, we use the rail profile UIC60 and two axial wheel of bogie H665. For example, FEM (a sixth of area) means the induced force in a sixth of elliptical contact surface that is equal to contact stress. Therefore, the results, with assumption of induced force in one-thirds of area are coincided to results of the hertz and new formulation methods. According to above information the pressure equal to $3.7 \times 10^{6} \mathrm{pa}$ is induced at the position of contact of wheel and axel. Comparisons of the results of five methods are presented in Table 1.

Figures 11, 12 and 13 show that the finite element results and modeling of the wheel and rail in the contact status $^{12}$.

According to the above results, exponential and power law formulations results are similar to the Hertz formulation, FEM and mentioned reference ${ }^{12}$ results. Eventually, we can rely on the present formulations. Also, in accordance with FEM results, critical regions are determined in the rail easily, (See Figures 12 and 13).

\section{Conclusions}

This study enabled us to calculate the magnitude of the expected elastic contact stresses and verify different critical area of the rail and wheel. The study also enabled

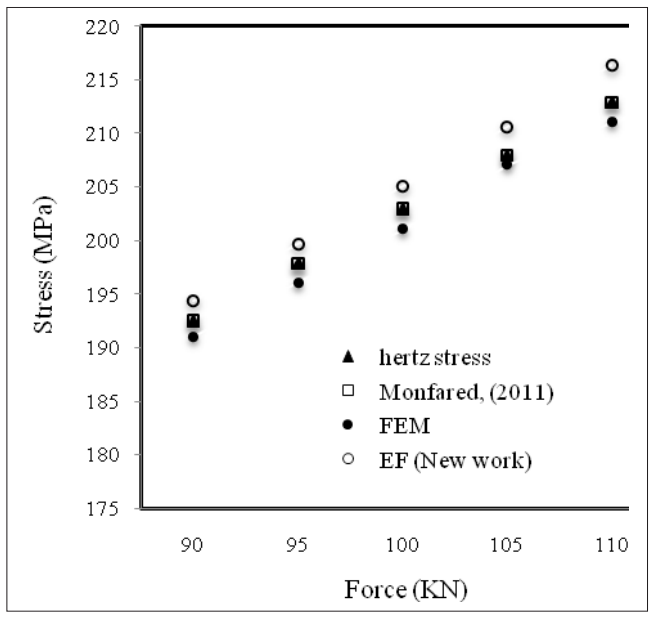

Figure 6. Comparisons of FEM (a quarter of area), hertz, new formulation ${ }^{12}$ and Exponential Form (EF) stresses.

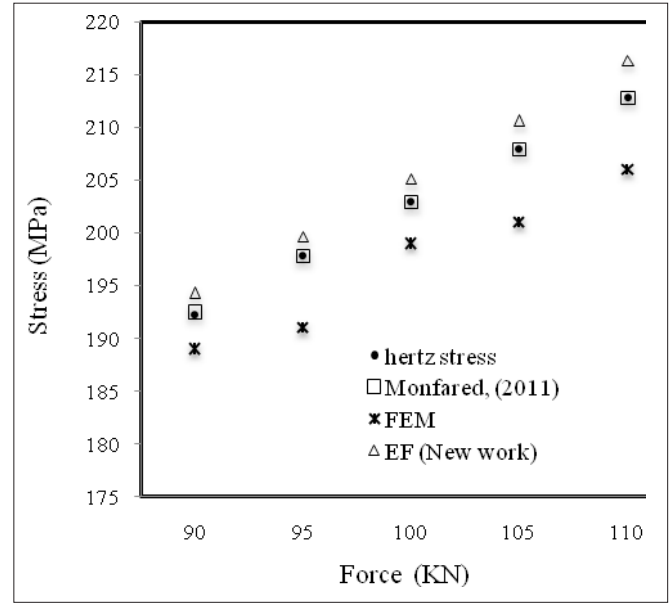

Figure 7. Comparisons of FEM (half of area), hertz, new formulation ${ }^{12}$ and Exponential Form (EF) stresses.

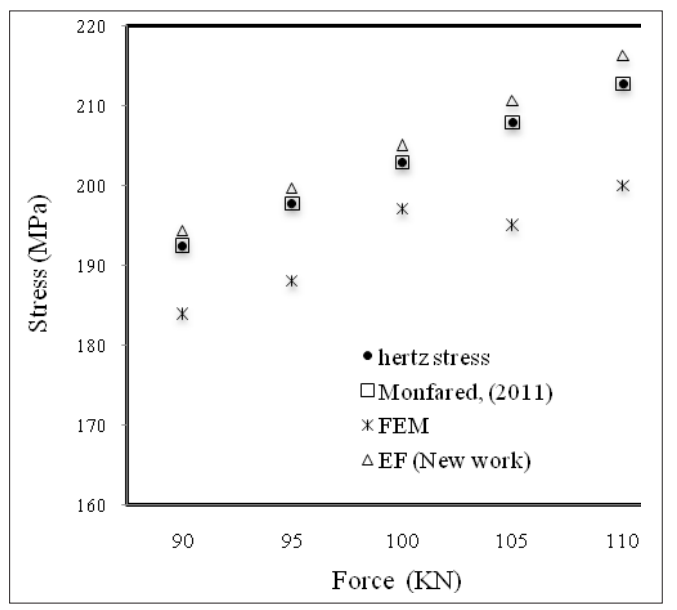

Figure 8. Comparisons of FEM (three-fourths of area), hertz stress, new formulation ${ }^{12}$ and Exponential Form (EF) stresses. 


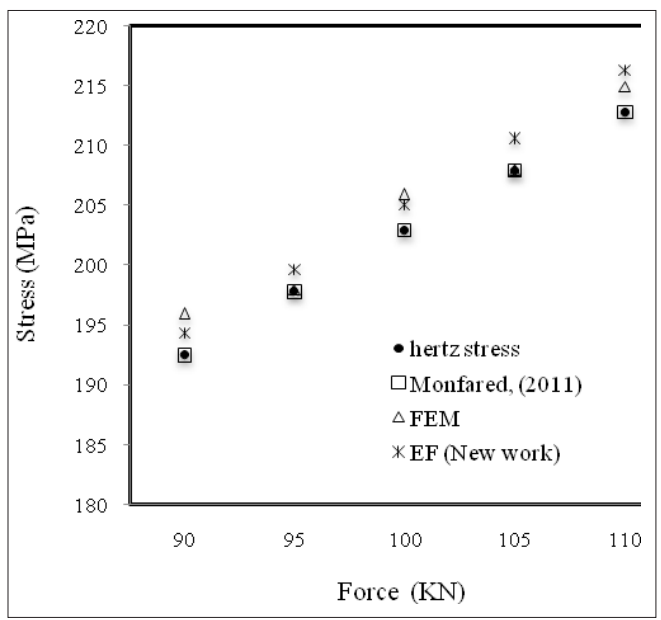

Figure 9. Comparisons of FEM (a sixth of area), hertz, new formulation $^{12}$ and Exponential Form (EF) stresses.

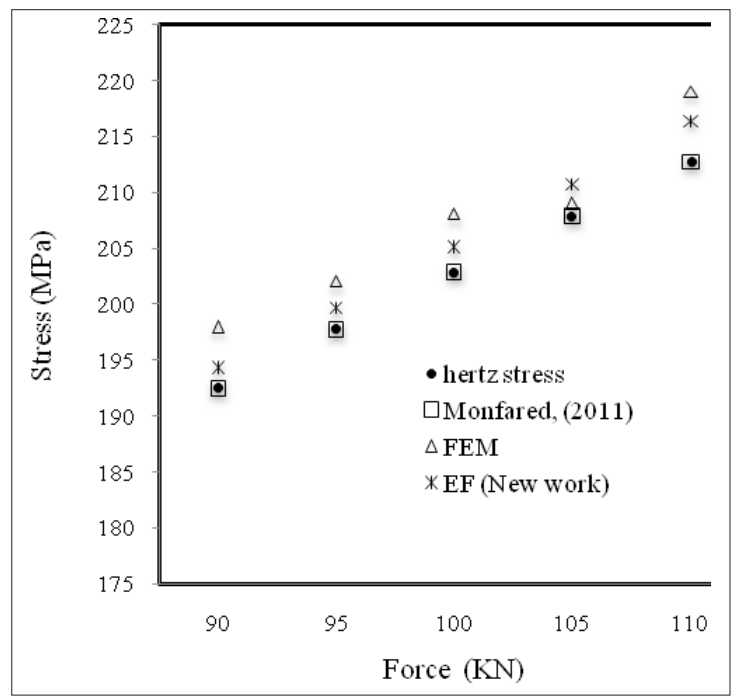

Figure 10. Comparisons of FEM (one-eighths of area), hertz, new formulation ${ }^{12}$ ) and Exponential Form (EF) stresses.

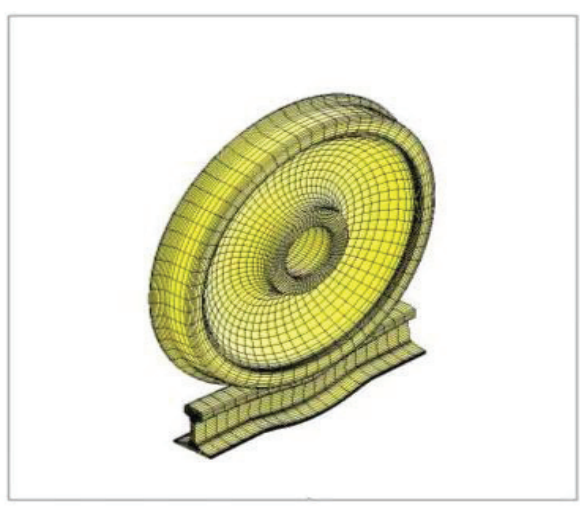

Figure 11. Position of contact of wheel and rail with FEM.

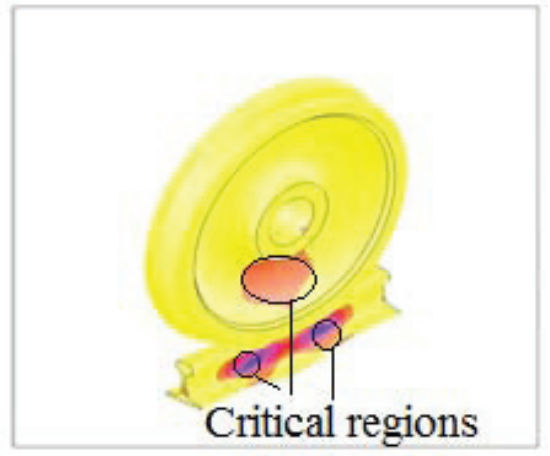

Figure 12. Von Mises stress in contact of wheel and rail with FEM.

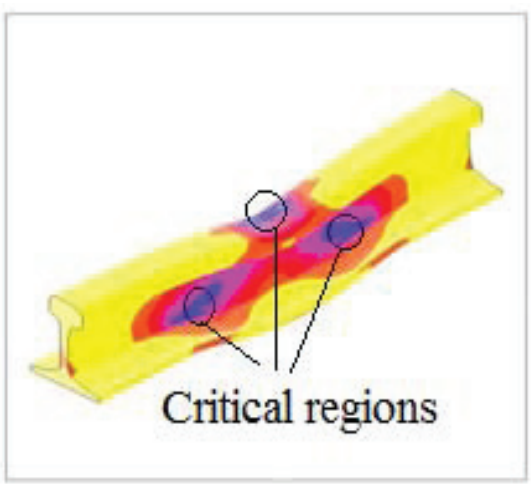

Figure 13. Equivalent stress in rail by FEM.

us to determine the critical stresses under high loading conditions in the rail and wheels. Numerical analysis of the stress-strain (FEM) specificities of 3D Rail-Wheel contact is done by FEM successfully. This contact problem is very nonlinear in its nature from the mechanic of materials view.

Also, this research shows an analytical formulation and manner of evaluating elastic contact stresses on contact rolling bodies for the case in which the contact area is elliptic. Results of the new contact stress formulations in exponential and power law forms (EF, PL) for rolling bodies are coincided with the results of the hertz stress, finite element method (FEM) and previous published analyses. Results, with assumption of induced and applied force in one-thirds of area are coincided with the results of the hertz and results of new exponential and power law formulations. Results of the Exponential and Power Law formulations (EF, PL) of the contact stress are similar to the hertz, FEM and prior published results. Finally, we can rely on this approach and their results. 


\section{References}

1. Smith JO, Liu CK. Stresses due to tangential and normal loads on an elastic solid with application to some contact stress problems. J. Appl. Mech. 1953; 20(2):157-166.

2. Haines DJ, Ollerton E. Contact stress distribution on elliptical contact surfaces subjected to radial and tangential forces Proc. Inst. Mech. Eng. 1963; 177(4):45-54.

3. Sackfield A, Hills DA. Some useful results in the classical Hertz contact problem. J. Strain. Anal. 1983; 18(2):101-105.

4. Ertz M, Knothe K. A comparison of analytical and numerical methods for the calculation of temperatures in wheel/ rail contact. Wear. 2002; 253(3-4):498-508.

5. Baek KS, Kyogoku K, Nakahara T. An experimental investigation of transient traction characteristics in rolling-sliding wheel/rail contacts under dry-wet conditions. Wear. 2007; 263(1-6):169-179.

6. Donzella G, Petrogalli C. A failure assessment diagram for components subjected to rolling contact loading. Int. J. Fatigue. 2010; 32(2):256-268.

7. Roviraa A, Rodaa A, Marshall MB, Brunskill H, Lewis R. Experimental and numerical modelling of wheel-rail contact and wear. Wear. 2011; 271:911-924.

8. Sladkowski A, Sitarz M. Analysis of wheel-rail interaction using FE software. Wear. 2005; 258:1217-1223.

9. Monfared V. Contact stress analysis in rolling bodies by Finite Element Method (FEM) Statically. J. Mech. Eng. Aut. 2011; 2(2):12-16.

10. Vasauskas V, Bazaras Ž, Čapas V. Strength anisotropy of railway wheels under contact load. Mechanika. 2005; 1(51):31-38.

11. Wen Z, Wu L, Li W, Jin X, Zhu M. Three-dimensional elastic-plastic stress analysis of wheel-rail rolling contact. Wear. 2011; 271:426-436.
12. Monfared V. A new analytical formulation for contact stress and prediction of crack propagation path in rolling bodies and comparing with finite element model (FEM) results statically. Int. J. Phys. Sci. 2011; 6(15): 3613-3618.

13. Arslan MA, Kayabasi O. 3-D Rail-Wheel contact analysis using FEA. Adv. Eng. Softw. 2012; 45:325-331.

14. Goodarzian H, Ghobadi M, Farahabadi MA, Mohammadnezhad $\mathrm{H}$, Hejazi SS. An investigation of nonlinear KdV type equations using HPM and VIM. Indian Journal of Science and Technology. 2011; 4:952-956.

15. Nikkhoo A, Amankhani M. Dynamic behavior of functionally graded beams traversed by a moving random load. Indian Journal of Science and Technology. 2012; 5(12):3727-3731.

16. Haghighi AR, Ghejlo HH, Asghari N. Explicit and implicit methods for fractional diffusion equations with the riesz fractional derivative. Indian Journal of Science and Technology. 2013; 6(7):4881-4885.

17. Srinivasan V. Analysis of static and dynamic load on hydrostatic bearing with variable viscosity and pressure. Indian Journal of Science and Technology. 2013; 6(6):4777-4782.

18. Anbazhagan R, Satheesh B, Gopalakrishnan K. Mathematical modeling and simulation of modern cars in the role of stability analysis. Indian Journal of Science and Technology. 2013; 6(5):4633-4641.

19. Loonker D, Banerji PK. Distributional dual series equations and fractional calculus. Indian Journal of Science and Technology. 2013; 6(1):3892-3897.

20. El-Marouf SAA. On some generalizations of the HilbertHardy type integral inequalities. Indian Journal of Science and Technology. 2013; 6(2):4098-4111. 